\title{
PREVALENCE AND MOLECULAR DETECTION OF METHICILLIN-RESISTANT STAPHYLOCOCCUS AUREUS FROM DOGS AND CATS IN DHAKA CITY
}

\author{
A. Habibullah ${ }^{1}$, A. M. M. T. Rahman ${ }^{2}$, M. R. Haydar ${ }^{1}$, K. H. M. N. H. Nazir ${ }^{1}$ and M. T. Rahman ${ }^{\text {1* }}$
}

${ }^{1}$ Department of Microbiology and Hygiene, Faculty of Veterinary Science, Bangladesh Agricultural University, Mymensingh-2202, Bangladesh; ${ }^{2}$ Adhunik Sodor Hospital, Naogaon, Bangladesh.

\begin{abstract}
Pet (dog and cat) animal numbers have substantially increased in modern society. There is concern over transmission of Staphylococcal infection including methicillin-resistant Staphylococcus aureus (MRSA) between animals and humans. The objective of the present study was to determine the prevalence of MRSA from apparently healthy and diseased dog and cat of different veterinary Hospital, clinics and pet animal market in Dhaka city. Samples collected for detection of MRSA were nasal swab, pus and wound swab. Among the 93 samples, $40.86 \%(\mathrm{n}=38 / 93)$ were confirmed as positive for $S$. aureus and $4.30 \%(\mathrm{n}=4 / 93)$ as MRSA. The detection of MRSA was confirmed phenotypically and also by PCR targeting mec Agene specific for MRSA. All isolates isolated $S$. aureus were coagulase positive and hence pathogenic. Antibiogram study showed that all these isolates were sensitive to vancomycin and tetracycline. The overall prevalence of MRSA was higher in dog (4.91\%) compared to cat $(3.13 \%)$. The highest prevalence of MRSA $(5.88 \%)$ was recorded in samples collected from K ataban Pet Animal Market, Dhaka. On sample basis MRSA was higher in nasal swab compared to pus and wound swab. On age basis, the prevalence of MRSA was higher in younger animal compared to older animal. The highest prevalence of $S$. aureus was found in diseased dog and highest prevalence of MRSA was found in diseased cat. None of the healthy cat was found positive for MRSA. Present study emphasizes that dogs and cats may act as a possible reservoirs for transmission of MRSA to human.
\end{abstract}

Keywords: Dog, cat, methicillin-resistant Staphylococcus aureus (MRSA), prevalence, mecA, PCR.

\section{INTRODUCTION}

Methicillin resistant Staphylococcus aureus (MRSA) is a specific strain of the S. aureus that has developed resistance against the beta-lactam class of antibiotics, which includes penicillin and derivates such as methicillin, oxacillin, and amoxicillin (Foster, 1996). The genetic basis of MRSA is due to the presence of mecA gene encoding low affinity penicillin-binding protein 2A (PBP2A), that act as a surrogate trans-peptidase in the presence of high concentrations of $\beta$-lactam antibiotics which inactivate the four high-affinity PBPs native to $S$. aureus (de Jonge and Tomasz, 1993).

MRSA was first discovered in the UK in 1961 but it is now widespread across the globe. Several studies have been done in Bangladesh especially in human to see the burden of MRSA infection (Haque et al. 2011; Islam et al., 2011) reported a prevalence of $43 \cdot 7 \%$ MRSA in clinical samples. In another study Islam et al. (2011) detected gene mecA responsible for resistance against methicillin by PCR from $25.0 \%$ human clinical samples. MRSA are also found in animal. In 1972, MRSA was found in milk from Belgian cows with mastitis. There are reports on the detection of MRSA from dogs, cats, sheep, cattle, horses, rabbits, seals, cockroach, guinea pig and chinchilla (Morgan, 2008; Chandrasekaran et al., 2014; Gulani et al., 2016; Islam et al., 2016). The mecA gene has been detected from Staphylococcus isolated from dairy cattle mastitis (Rahman et al., 2005).

MRSA are serious public health concern since they could not be treated effectively with many antibiotics easily. Lee et al. (2003) reported the potentiality of transmission of MRSA from food animal to human. Across the globe, an estimated 2 billion people carry some form of $S$. aureus; of these, up to 53 million $(2.7 \%$ of carriers) are thought to carry MRSA. It has been suggested that zoonotic transmission of MRSA can takes occur between pet owners and their pets (VAN Balen et al., 2017). Several works have been carried out in abroad on the prevalence of MRSA in dog and cat (VAN Balen et al., 2017; Karkaba et al., 2017). In Bangladesh, MRSA has been isolated from raw milk samples of cow (Jahan et al., 2015). However, no work has been reported in Bangladesh describing the isolation of MRSA from healthy and diseased Dog and Cat in Dhaka city. In the present study, we investigated the prevalence of MRSA in dog and cat used as pet animal in Dhaka city and their zoonotic significance.

*Corresponding e-mail address: tanvirahman@bau.edu.bd Copyright $@ 2017$ Bangladesh Society for Veterinary Medicine 


\section{A. Habibullah and others}

\section{MATERIALS AND METHODS}

\section{Sampling areas}

The pet dogs and cats used for this study were available at the Central Veterinary Hospital (CVH), Dhaka; Gulshan Pet Clinic, Dhaka; Kataban Pet Animal Market, Dhaka. Dhaka, the capital city of Bangladesh.

\section{Collection of samples}

Nasal swab, pus and wound swab sample were collected from dog and cat pet clinics. A total of 93 swab samples were collected. All of these pets were Hybrid and cross breed with average age of 1-3 years old. Detail of the sampling data is available in Table 1.

Table 1. Name of collection points with the number of samples collected from each of the points

\begin{tabular}{|c|c|c|c|c|c|}
\hline \multirow{3}{*}{$\begin{array}{l}\text { Sample } \\
\text { collection area }\end{array}$} & \multicolumn{4}{|c|}{ No. of samples } & \multirow{3}{*}{$\begin{array}{l}\text { Total } \\
\text { Dog and Cat }\end{array}$} \\
\hline & \multicolumn{2}{|l|}{ Dog } & \multicolumn{2}{|l|}{ Cat } & \\
\hline & Healthy & Diseased & Healthy & Diseased & \\
\hline $\begin{array}{l}\text { Central Veterinary } \text { Hospital } \\
(\mathrm{CVH}), \text { Dhaka }\end{array}$ & 13 & 34 & 07 & 12 & 66 \\
\hline $\begin{array}{l}\text { Kataban Pet Animal Market, } \\
\text { Dhaka }\end{array}$ & 08 & 02 & 06 & 01 & 17 \\
\hline Gulshan Pet Clinic, Dhaka & 01 & 03 & 02 & 04 & 10 \\
\hline Diseased or Healthy & 22 & 39 & 15 & 17 & 93 \\
\hline
\end{tabular}

\section{Isolation of Staphylococcus aureus}

Isolation of $S$. aureus was done using standard culture, staining and biochemical test, as described by Islam et al. (2007) and Jahan et al. (2015).

\section{PCR for detection of mec $A$ gene}

The genomic DNA from S. aureus was extracted by boiling method as described by Begum et al. (2016). Detection of mecA was done by PCR using primers (Table 2) described by Bennimath et al. (2011).

Each PCR was done in $25 \mu \mathrm{l}$ reaction that consisted of $5 \mu \mathrm{l}$ genomic DNA, $12.5 \mu \mathrm{l}$ PCR master mixture (Promega) $1 \mu \mathrm{l}$ of each of the two primers and $5.5 \mu \mathrm{l}$ of nuclease free water. The PCR amplification was done by initial denaturation at $95^{\circ} \mathrm{C}$ for 5 minutes, followed by 30 cycles of denaturation at $95^{\circ} \mathrm{C}$ for 1 minute, annealing temperature of primers was $55^{\circ} \mathrm{C}$ for 45 seconds and extension at $72^{\circ} \mathrm{C}$ for 1.5 minutes. The final extension was conducted at $72^{\circ} \mathrm{C}$ for 3 minutes. At the end of PCR, the PCR produces were run on a $2 \%$ agarose gel, stained with ethidium bromide and photographed using a Gel documentation system (BioRad).

Table 2. Primers used in PCR for the detection of methicillin resistant S. aureus

\begin{tabular}{llll}
\hline Primer name & Primers sequence & Product size $(\mathrm{bp})$ & Reference \\
\hline$m e c A / \mathrm{F}$ & 5'-GTGGAATTGGGCAATACACC-3' & 533 & $\begin{array}{l}\text { Bennimath } \\
\text { et al. }(2011)\end{array}$ \\
\hline mecA/R & 5'-AGTTCTGCAGTACCGGAT -3' & & \\
\hline
\end{tabular}

Antibiotic sensitivity test

Antibiotic sensitivity test of the isolated $S$. aureus was performed using Mueller Hinton agar as described by Kirby-Bauer disc diffusion method (Begum et al., 2016). Inhibition of zone diameters was measured and values obtained from the National Committee on Clinical Laboratory Standards were used to interpret the results obtained. S. aureus isolates were then classified as resistant, intermediate resistant or susceptible to a particular antibiotic based on the standard interpretation table updated according to the Clinical and Laboratory Standards Institute (CLSI) guidelines (CLSI, 2011). The antibiotics disc used were methicillin (oxacillin) $-10 \mu \mathrm{g} / \mathrm{disc}$, vancomycin-30 $\mu \mathrm{g} /$ disc and tetracycline $30 \mu \mathrm{g} /$ disc. 


\section{RESULTS}

Prevalence and molecular detection of methicillin

A total of 93 samples were collected and examined for isolation and identification of $S$. aureus to detect MRSA. Out of the 93 swab samples, $38(40.86 \%)$ isolates were identified as $S$. aureus on the basis of morphology, staining and cultural characteristics on blood agar (BA) and Mannitol Salt Agar (MSA). Grams staining identified them as grape like clusters. In BA they produced characteristics $\beta$-hemolysis (Figure 1) and on MSA they fermented mannitol and produced characteristics yellowish colonies (Figure 2).

\section{Detection of MRSA based on phenotype}

Isolated $S$. aureus were subjected to antimicrobial resistance profile assessment for phenotypic investigation of MRSA. MRSA is identified by assessing zone of inhibitions with oxacillin $\leq 10 \mathrm{~mm}$ (CLSI, 2011) (Figure 3). Among the $38 \mathrm{~S}$. aureus 4 was found resistant to oxacillin (methicillin).

\section{Confirmation of MRSA by PCR targeting mecA gene}

In order to develop a quick and reliable confirmatory diagnostic test using molecular method for MRSA PCR was performed. At the genomic level, the optimized PCR assay was able successfully to amplify the target mecA gene expected size $533 \mathrm{bp}$ fragment from the genomic DNA. The presence of methicillin resistant $S$. aureus was confirmed in two samples by amplification of the approximately $533 \mathrm{bp}$ DNA fragment (Figure 4).

\section{Prevalence of Staphylococcus aureus and MRSA in dog and cat}

The overall prevalence of $S$. aureus was higher in dog (42.62\%) compared to cat $(37.50 \%)$ (Table 3, Figure 5). Similarly the overall prevalence of MRSA was higher in dog (4.91\%) compared to cat $(3.13 \%)$ (Table 3).Prevalence of $S$. aureus in healthy dog and cat was $22.73 \%$ and $26.67 \%$ respectively, where diseased dog and cat was $53.85 \%$ and $47.05 \%$ respectively (Table 3 ). The prevalence of MRSA in healthy dog was $4.55 \%$ while none of the healthy cat was found positive for MRSA. The prevalence of MRSA in diseased dog and cat was $5.13 \%$ and $5.88 \%$ respectively. The highest prevalence of $S$. aureus was found in diseased dog and highest prevalence of MRSA was found in diseased cat (Table 4).

Table 3. Prevalence of $S$. aureus and MRSA between dog and cat

\begin{tabular}{ccccc}
\hline Species & Tested sample & No. of S. aureus (\%) & No. of positive MRSA & Prevalence of MRSA (\%) \\
\hline Dog & 61 & $26(42.62 \%)$ & 3 & $4.91 \%$ \\
\hline Cat & 32 & $12(37.50 \%)$ & 1 & $3.13 \%$ \\
\hline Total & 93 & $38(40.86 \%)$ & 4 & $4.30 \%$ \\
\hline
\end{tabular}

Table 4. Prevalence of Staphylococcus aureus and MRSA in dog and cat according to health status

\begin{tabular}{llcccc}
\hline \multicolumn{2}{l}{ Species } & Tested sample & No. of S. aureus $(\%)$ & No. of positive MRSA & Prevalence of MRSA (\%) \\
\hline \multirow{3}{*}{ Dog } & Healthy & 22 & $5(22.73 \%)$ & 1 & $4.55 \%$ \\
\cline { 2 - 6 } & Diseased & 39 & $21(53.85 \%)$ & 2 & $5.13 \%$ \\
\hline \multirow{2}{*}{ Cat } & Healthy & 15 & $4(26.67 \%)$ & 0 & $0.00 \%$ \\
\cline { 2 - 6 } & Diseased & 17 & $8(47.05 \%)$ & 1 & $5.88 \%$ \\
\hline Total & & 93 & $38(40.86 \%)$ & 4 & $4.30 \%$ \\
\hline
\end{tabular}

\section{Prevalence of $\boldsymbol{S}$. aureus and MRSA according to type of samples}

Samples analyzed in this study were nasal swab, wound swab and pus. Prevalence of $S$. aureus and MRSA in nasal swab, wound swab, pus was $30.99 \%, 64.28 \%, 87.5 \%$ and $4.23 \%, 0.00 \%, 12.5 \%$ respectively (Table 5).

\section{Prevalence of $S$. aureus and MRSA in dog and cat according to source of samples}

The prevalence of $S$. aureus and MRSA based on source of samples of Dhaka city is shown in Table 6. Highest prevalence of $S$. aureus was found in Central Veterinary Hospital (CVH), Dhaka (46.97\%). Prevalence of $S$. aureus in Kataban Pet Animal Market, Dhaka and Gulshan Pet Clinic, Dhaka were (23.53\%) and (30\%) respectively. The highest prevalence of MRSA was recorded Kataban Pet Animal Market, Dhaka (5.88\%) 


\section{A. Habibullah and others}

compared to Central Veterinary Hospital (CVH), Dhaka (4.55\%) Kand Gulshan Pet Clinic, Dhaka $(0.00 \%)$ shown in Table 11.

Table 5. Prevalence of S. aureus and MRSA indifferent types of samples

\begin{tabular}{|c|c|c|c|c|}
\hline \multirow[t]{2}{*}{ Collected samples } & \multicolumn{2}{|c|}{ Total samples $(n=93)$} & \multirow{2}{*}{$\begin{array}{c}\text { Total no. of positive } S \text {. aureus } \\
(\mathrm{n}=38)\end{array}$} & \multirow{2}{*}{$\begin{array}{l}\text { Total no. of positive } \\
\text { MRSA }(n=4)\end{array}$} \\
\hline & $\operatorname{Dog}(61)$ & Cat (32) & & \\
\hline Nasal swab & $47(77.04 \%)$ & $24(75 \%)$ & $22(30.99 \%)$ & $3(4.23 \%)$ \\
\hline Wound swab & $9(14.75 \%)$ & $5(15.63 \%)$ & $9(64.28 \%)$ & $0(0.00 \%)$ \\
\hline Pus & $5(8.20 \%)$ & $3(9.38 \%)$ & $7(87.5 \%)$ & $1(12.5 \%)$ \\
\hline
\end{tabular}

Table 6. Prevalence of $S$. aureus and MRSA in dog and cat according to source of samples

\begin{tabular}{lccccc}
\hline Sample collection area & $\begin{array}{l}\text { Tested } \\
\text { sample }\end{array}$ & $\begin{array}{l}\text { No. of positive } \\
\text { S. aureus }(\%)\end{array}$ & $\begin{array}{l}\text { No. of positive } \\
\text { MRSA }\end{array}$ & $\begin{array}{l}\text { Prevalence of } \\
\text { MRSA }(\%)\end{array}$ \\
\hline $\begin{array}{l}\text { Central Veterinary } \\
\text { (CVH), Dhaka }\end{array}$ & Hospital & 66 & $31(46.97 \%)$ & 3 & $4.55 \%$ \\
\hline $\begin{array}{l}\text { Kataban Pet Animal } \\
\text { Dhaka }\end{array}$ & Market, & 17 & $4(23.53 \%)$ & 1 & $5.88 \%$ \\
\hline Gulshan Pet Clinic, Dhaka & 10 & $3(30 \%)$ & 0 & $0.00 \%$ \\
\hline \multicolumn{2}{l}{\begin{tabular}{l} 
Total \\
\hline
\end{tabular}}
\end{tabular}

\section{Prevalence of $S$. aureus and MRSA in dog and cat according to age}

Prevalence of $S$. aureus and MRSA in different age of dog and cat are shown in Table 7. Prevalence of $S$. aureus was highest in $\geq 1.5$ year dog $42.86 \%$ than $<1.5$ year dog $42.42 \%$ and prevalence of MRSA in $<1.5$ year $\operatorname{dog}$ was $6.06 \%$, which was higher than $\geq 1.5$ year $\operatorname{dog} 3.57 \%$. On the other hand, Prevalence of $S$. aureus was highest in $<1$ year cat $42.10 \%$ than $\geq 1$ year cat $30.77 \%$ and prevalence of MRSA in $<1$ year cat was $5.26 \%$, which was higher than $\geq 1$ year cat $0.00 \%$.

Table 7. Age wise prevalence of $S$. aureus and MRSA in dog and cat

\begin{tabular}{lccccc}
\hline Age & & $\begin{array}{l}\text { Tested } \\
\text { sample }\end{array}$ & $\begin{array}{l}\text { No. of } \\
\text { S. aureus }(\%)\end{array}$ & $\begin{array}{l}\text { No. of positive } \\
\text { MRSA }\end{array}$ & $\begin{array}{l}\text { Prevalence of } \\
\text { MRSA (\%) }\end{array}$ \\
\hline \multirow{2}{*}{ Dog } & $\geq 1.5$ year & 28 & $12(42.86 \%)$ & 1 & $3.57 \%$ \\
& $<1.5$ year & 33 & $14(42.42 \%)$ & 2 & $6.06 \%$ \\
\hline \multirow{2}{*}{ Cat } & $\geq 1$ year & 13 & $4(30.77 \%)$ & 0 & $0.00 \%$ \\
& $<1$ year & 19 & $8(42.10 \%)$ & 1 & $5.26 \%$ \\
\hline Total & & 93 & $38(40.86 \%)$ & 4 & $4.30 \%$ \\
\hline
\end{tabular}

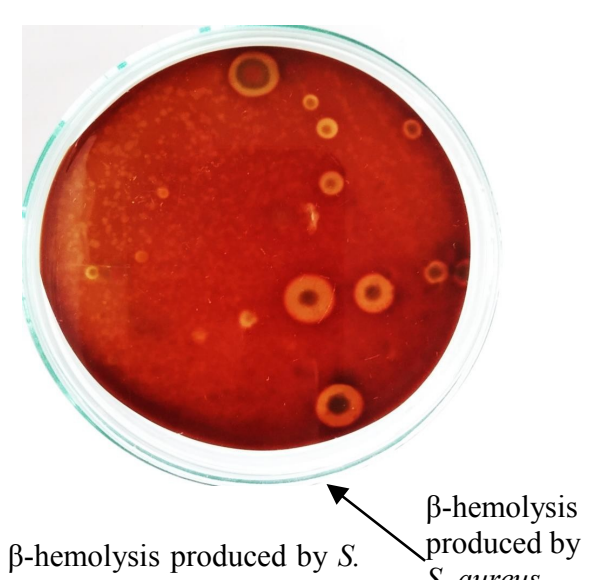

Figure 1. $\beta$-hemolysis produced by $S$ S. aureus qureus on $5 \%$ sheep blood agar 8

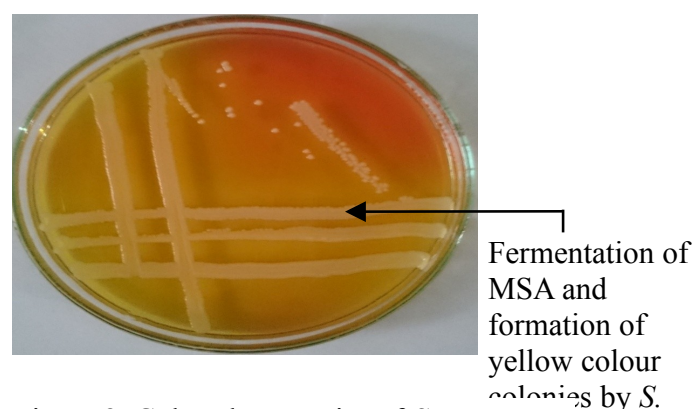

Figure 2. Cultural properties of $S$. aureus onto MSA media 


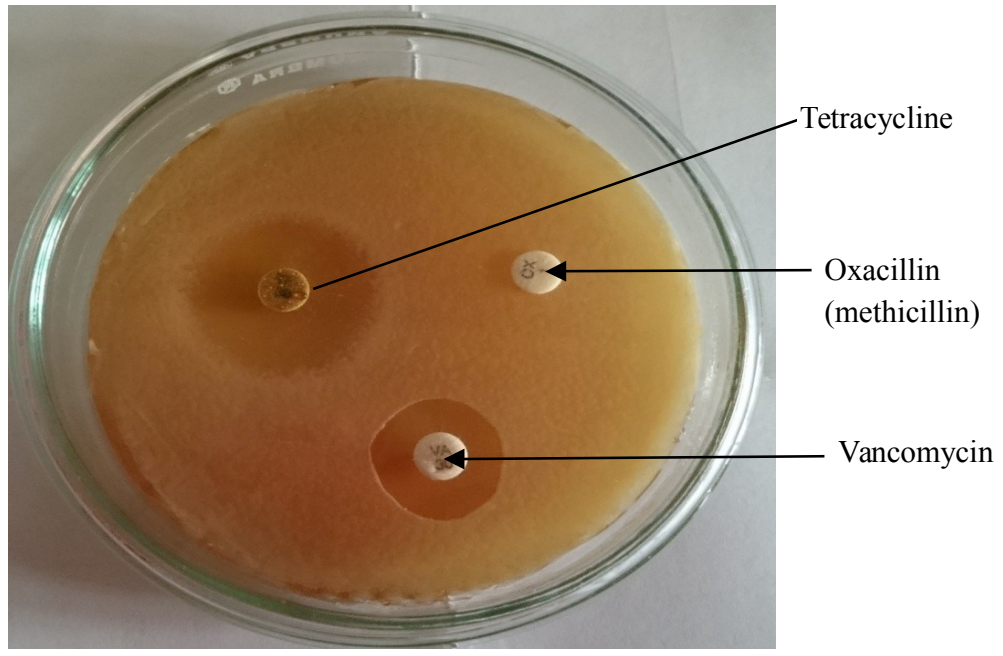

Figure 3. Methicillin (oxacillin), vancomycin and tetracycline sensitive pattern of S. aureus

\section{3}

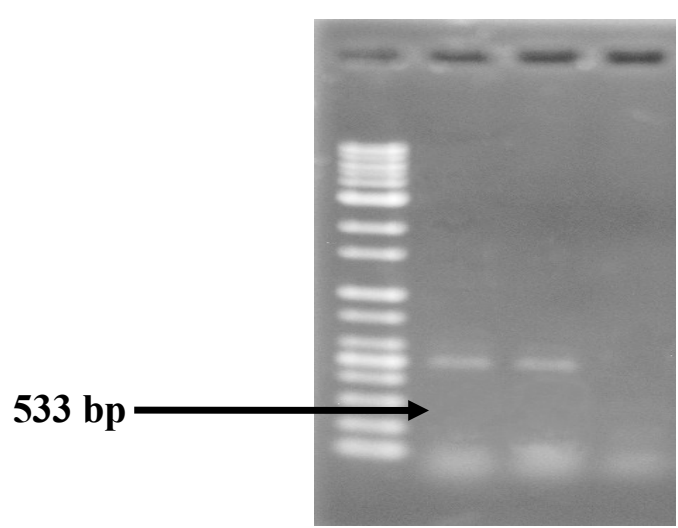

Figure 4. Molecular detection of methicillin resistant Staphylococcus aurous by PCR

(Lane 1: 1kb DNA ladder, Lanes 2-3: Tested samples, Lane 4: Negative control)

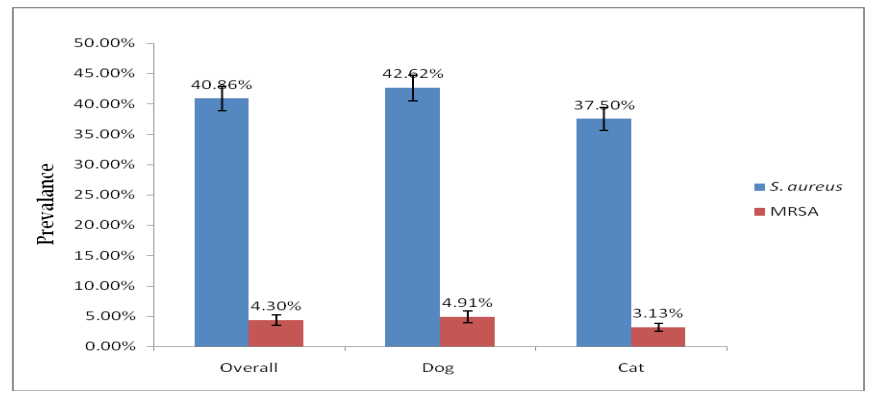

Figure 5. The overall prevalence of $S$. aureus and MRSA in dog and cat 


\section{A. Habibullah and others}

\section{DISCUSSION}

MRSA has emerged as a significant public health problem both in human and veterinary medicine worldwide. Among the Gram-positive pathogens, S. aureus continues to cause skin and soft tissue infections in human and animal including hospitalized patients (Joshi et al., 2011). MRSA got zoonotic importance when scientists suggested the possibility of dog and cat serving as reservoirs for human MRSA infection.

In this study, we investigated the prevalence MRSA in pet dogs and cats of Dhaka city. Among the 93 samples analyzed (61 from pet dogs and 32 from pet cats) 38 (40.86\%) was found positive for S. aureus. All these 38 isolates were considered as pathogenic since all of them were coagulase positive, although many of these were isolated from healthy animal. Among these $38 \mathrm{~S}$. aureus, four (4.30\%) isolates were found to show resistant to oxacillin and considered as MRSA. But interestingly all these isolates were found sensitive to vancomycin and tetracycline, suggesting that vancomyc in and tetracycline could be the preferred antibiotic to treat the infection caused by these $S$. aureus including the MRSA. In fact previously Oberoi et al. (2012) and Lee (2003) also showed the susceptible of MRSA to vancomycin. But Tiwari and Sen (2006) reported resistance of MRSA against vancomycin resistance. According to a conference report submitted by Rahman (2015) the prevalence of MRSA was found $5.12 \%$ in Dog in Dhaka city, which is similar to the finding we have reported here.

The highest prevalence of MRSA was recorded Kataban Pet Animal Market, Dhaka (5.88\%). People bring their pet in the Kataban Pet Animal Market for sell. It was not unexpected to see the highest prevalence of MRSA in pet in this market, because it is a mixing place of different types of animal, allowing cross contamination of MRSA positive animal with MRSA negative animals.

To the best of our knowledge, this is the first report describing prevalence of MRSA in healthy and disease Dog and Cat in Dhaka city in Bangladesh. Presence of MRSA in pet animal as detected in this study is alarming, because MRSA are zoonotic in nature. The pet may act as potential reservoirs for transmission for MRSA to human. Attention therefore should be taken for early detection of MRSA and application of suitable antibiotic to cure them.

\section{CONCLUSIONS}

MRSA is a health problem both for animal and human across the globe. Pets (dog and cat) animal may act as a reservoir and source of MRSA for human. In this study we determined the prevalence of MRSA in apparently healthy and diseased dog and cat in Dhaka city. Among the 93 samples, $40.86 \%$ ( $=38 / 93)$ were confirmed as positive for $S$. aureus and $4.30 \%(\mathrm{n}=4 / 93)$ for MRSA. All $S$. aureus isolates were pathogenic in nature as revealed by coagulase test. Present study suggests that dogs and cats may act as possible reservoirs for transmission of MRSA to human.

\section{ACKNOWLEDGEMENTS}

The researchers would like to thank Central Veterinary Hospital (CVH), Dhaka, Kataban Pet Animal Market, Dhaka and Gulshan Pet Clinic, Dhaka for providing the samples and data.

\section{REFERENCES}

1. Begum F, Islam MM, Sohidullah MM, Kabir SML, Islam M and Rahman MT (2016). Molecular identification and antibiogram profiles of Escherichia coli isolated from apparently healthy and diarrheic goats. Bangladesh Journal of Veterinary Medicine 14: 203-208.

2. Chandrasekaran D, Venkatesan P, Tirumurugaan KG, Gowri B, Subapriya S and Thirunavukkarasu S (2014). Subacute mastitis associated with Methicillin Resistant Staphylococcus aureus in a cow: A case report. Journal of Advanced Veterinary and Animal Research 1: 235-237.

3. CLSI (2011). Performance Standards for Antimicrobial susceptibility Testing; Twenty-First Informational Supplement.30 (1).Clinical Lab. Standard Ins. USA.

4. deJonge BLM and Tomasz A (1993). Abnormal peptidoglycan produced in a methicillin-resistant strain of Staphylococcus aureus grown in the presence of methicillin: functional role for penicillin-binding protein $2 \mathrm{~A}$ in cell wall synthesis. Antimicrobial Agents and Chemotherapy 37: 342-346. 
5. Foster T (1996). Staphylococcus. In: Barron's Medical Microbiology (Barron S et al.,eds.), 4th ed., Univ. of Texas Medical Branch.

6. Gulani IA, Geidam YA, Adamu L, Lawal JR and Abadam FA (2016). Prevalence and phenotypic detection of methicillin resistance Staphylococcus aureus between ruminants butchered for humanoid intake and animal handlers in Maiduguri, Nigeria. Journal of Advanced Veterinary and Animal Research 3: 152-159.

7. Haque N, Bari MS, Bilkis L, Haque S and Sultana S (2011). Methicillin-Resistant Staphylococcus aureus. Mymensingh Medical Journal 20: 59-64.

8. Islam A, Nath $\mathrm{AD}$, Islam $\mathrm{K}$, Islam $\mathrm{S}$, Chakma $\mathrm{S}$, Hossain MB, Al-Faruq A and Hassan MM (2016). Isolation, identification and antimicrobial resistance profile of Staphylococcus aureus in Cockroaches (Periplaneta americana). Journal of Advanced Veterinary and Animal Research 3: 221-228.

9. Islam MA, Alam MM, Uddin MS, Kobayashi N and Ahmed MU (2011). Detection of Methicillin-Resistant Staphylococcus aureus (MRSA) from Animal and human origin in Bangladesh by Polymerase Chain Reaction. Bangladesh Journal of Veterinary Medicine 9: 161-166.

10. Islam MJ, Uddin MS, Islam MS, Nasrin MS, Nazir KHMNH, Rahman MT and Alam MM (2007). Prevalence of enterotoxigenic and toxic shock syndrome toxin-1 producing coagulase-positive Staphylococcus aureus in human and their characterization. Bangladesh Journal of Veterinary Medicine 5: 115-119.

11. Jahan M,Rahman M, Parvej MS, Chowdhury SMZH, Haque ME, Talukder MAK and Ahmed S (2015). Isolation and characterization of Staphylococcus aureus from raw cow milk in Bangladesh. Journal of Advanced Veterinary and Animal Research 2: 49-55.

12. Joshi A, Van de Peer Y and Michoel T (2011). Structural and functional organization of RNA regulons in the posttranscriptional regulatory network of yeast. Nucleic Acids Research 39: 9108-9117.

13. Karkaba A, Benschop J, Hill KE and Grinberg A (2017). Characterisation of methicillin-resistant Staphylococcus aureus clinical isolates from animals in New Zealand, 2012-2013, and subclinical colonisation in dogs and cats in Auckland. New Zealand Veterinary Journal 65: 78-83.

14. Lee JH (2003). Methicillin (oxacillin)-resistant Staphylococcus aureus strains isolated from major food animals and their potential transmission to humans. Applied and Environmental Microbiology 69: 6489-6494.

15. Morgan M (2008). Methicillin-resistant Staphylococcus aureus and animals: zoonosis or humanosis? Journal of Antimicrobial Chemotherapy 62: 1181-1187.

16. Oberoi L, Kaur R and Aggarwal A (2012). Prevalence an antimicrobial susceptibility pattern of Methicillin resistant Staphylococcus aureus (MRSA) in a rural tertiary care hospital in north India. International Journal of Applied Biology and Pharmaceutical Technology 3: 200-205.

17. Rahman M (2015). 3rd International Congress on Bacteriology and Infectious Diseases August 04-06, 2015 Valencia, Spain.

18. Rahman MT, Kobayashi N and Alam MM (2005). Genetic analysis of mecA homologues in Staphylococcus sciuri strains derived from mastitis in dairy cattle. Microbial Drug Resistance 11: 205-214.

19. Tiwari SK and Sen MR (2006). Emergence of vancomycin resistant Staphylococcus aureus (VRSA) from a tertiary care hospital from northern part of India. BMC Infectious Disease 6: 156.

20. VAN Balen JC, Landers T, Nutt E, Dent A and Hoet AE (2017). Molecular epidemiological analysis to assess the influence of pet-ownership in the biodiversity of Staphylococcus aureus and MRSA in dog- and non-dog-owning healthy households. Epidemiology and Infection 145: 1135-1147. 